Instituto Internacional de Investigación y Desarrollo Tecnológico Educativo INDTEC, C.A.

DOI: https://doi.org/10.29394/Scientific.issn.2542-2987.2019.4.12.6.127-140

OAI-PMH: http://www.indteca.com/ojs/index.php/Revista Scientific/oai

Artículo Original / Original Article

\title{
Elevar el Rendimiento Académico con Estrategias Educativas
}

\author{
Autora: Lenny Yesenia Rojas Suárez \\ Universidad Pedagógica Experimental Libertador, UPEL \\ lennyrojas810@gmail.com \\ Barinas, Venezuela \\ https://orcid.org/0000-0003-1102-991X
}

\section{Resumen}

El propósito de este estudio consiste en proponer las estrategias educativas innovadoras, como herramienta para elevar el rendimiento académico, en la Escuela Estadal Bolivariana "Miguel Tobías Quiñonez", del municipio Barinas, Estado Barinas, las cuales se derivan de un diagnóstico previo que arrojó poco rendimiento en los estudiantes de la institución. Las variables en que se apoya esta investigación son: rendimiento académico y estrategias educativas. El tipo de investigación es de Proyecto Factible el cual persigue diseñar un plan con el fin de mejorar la situación evidenciada, esta investigación se orienta en un estudio de campo, en la cual se obtendrán los datos directamente de la realidad, el enfoque es cuantitativo, lo que permite el uso y aplicación de cuestionarios para la obtención de la información. La población estará constituida por (07) docentes, que constituye la muestra. La validez del instrumento será obtenida mediante un juicio de expertos, quienes evaluarán la pertinencia, claridad, coherencia y redacción de las hojas de trabajo a aplicar. Los datos fueron compilados y examinados mediante la información obtenida a través de los instrumentos empleados. El estudio de los resultados permitirá conocer que en la institución se debe innovar desarrollando un plan basado en estrategias educativas para mejorar el rendimiento académico.

Palabras clave: innovación educacional; estrategias educativas; rendimiento académico. 


\title{
Raise Academic Performance with Innovative Activities
}

\begin{abstract}
The purpose of this study is to propose innovative educational strategies, as a tool to raise academic performance, in the Bolivarian State School "Miguel Tobías Quiñonez", Barinas municipality, Barinas State, which are derived from a previous diagnosis that showed little performance in the students of the institution. The variables on which this research is based are: academic performance and educational strategies. The type of research is Project Feasible which aims to design a plan in order to improve the situation evidenced, this research is oriented in a field study, in which the data will be obtained directly from reality, the approach is quantitative, which allows the use and application of questionnaires to obtain the information. The population will be constituted by (07) teachers, which constitutes the sample. The validity of the instrument will be obtained through an expert judgment, who will evaluate the relevance, clarity, coherence and writing of the worksheets to be applied. The data was compiled and examined through the information obtained through the instruments used. The study of the results will allow knowing that the institution must innovate by developing a plan based on educational strategies to improve academic performance.
\end{abstract}

Keywords: educational innovation; educational strategies; academic achievement. 


\section{Introducción}

En todo proyecto de aprendizaje que se desarrolle en un aula de clase es imprescindible el desarrollo de actividades, las cuales son apoyadas con estrategias educativas que se deben aplicar y adecuar al contexto educativo actual y a las necesidades de los estudiantes; la motivación y el interés que el docente debe poseer y las estrategias educativas e innovadoras que debe aplicar en su praxis se deben orientar hacia la integración, participación y producción activa del alumno para el desarrollo de su conocimiento, elevando así su aprendizaje.

El docente debe trazarse objetivos de cómo motivar al alumno a que se incorpore a su clase, qué estrategias educativas e innovadoras puede aplicar para que desde su praxis el docente impulse al alumno para que este mejore su rendimiento y nivel académico. Estos aspectos aún no están resueltos en la Escuela Estadal Bolivariana "Miguel Tobías Quiñonez". Las respuestas dependerán de la conducta, motivación y el enfoque psicológico que adopten. Por esta razón, el rendimiento académico, funge como elemento principal y depende del compromiso y de la calidad de los docentes.

Según Nieto (2008), expresa que: "los alumnos con menor rendimiento académico proceden de grupos familiares con el menor nivel económico y más bajo nivel de estudios de su familia" (pág. 264); reconociendo que en estos casos la función del docente tiene un papel muy importante para el alcance cognoscitivo del estudiante. Sin embargo; en algunos casos en las escuelas se cuenta con niños y niñas que no cuentan con el nivel académico correspondiente al grado en el que están y es allí donde surgen algunas variables las cuales se debe estudiar para detectar los elementos que influyen en el índice académico.

Por su parte, al implementar este plan, brinda el apoyo en aquellos estudiantes que presentan ciertas debilidades en desarrollar sus actividades pedagógicas facilitando su participación en la jornada liberadora; puesto que 
el dinamismo, la interacción y adaptación de los estudiantes en un ambiente innovador con un nuevo contexto escolar, se puede mejorar los bajos índices del rendimiento escolar, tomando en cuenta que innovando es hacer que la persona piense críticamente cambiando el contexto actual por uno mejor, por lo que al intercambiar ideas se crea un conocimiento productivo mejorando así las relaciones interpersonales en entre docentes y alumnos.

Es decir; la innovación no depende solo de la aplicación de estrategias educativas o las tradicionales, sino depende del clima participativo, creativo y del protagonismo que tome el alumno para la construcción de su conocimiento ya que creando y haciendo se aprende más. De esta manera se propicia y es un punto de partida para que cada uno establezca oportunamente los cambios necesarios y suficientes atribuyendo estrategias educativas e innovadoras.

En muchas Instituciones educativas, sobre todo en la parte rural, ha venido observándose poca utilización de estrategias educativas que motiven al alumno para mejorar su rendimiento académico y así disminuir la deserción escolar o en otros casos la repitencia de grados, la escuela Estadal Bolivariana "Miguel Tobías Quiñonez", no escapa a esta realidad por lo que la autora se formula la propuesta de estrategias pedagógicas innovadoras, basadas en un plan como herramienta para elevar el índice académico en los alumnos de esta institución antes mencionada, con el fin de desarrollar cognoscitivamente las habilidades de los estudiantes disminuyendo el fracaso escolar o deserción.

La función de los docentes toma un rol primordial dentro de los ambientes educativos porque contribuye en que las aulas se conviertan en espacios activos y productivos, generadores de nuevas ideas y aprendizajes, donde evidentemente sigan siendo promotores de valores, convivencia y ciudadanía; dando así un auge pedagógico para las diferentes obligaciones y curiosidades de los estudiantes facilitando las respuestas precisas a las exigencias de los estudiantes; es así que se hace necesario plantear algunas 
interrogantes que orienten mejor la investigación.

¿Es de importancia diagnosticar la rasante competencias que poseen los dicentes de la Escuela Estadal Bolivariana "Miguel Tobías Quiñonez" del municipio Barinas estado Barinas?; ¿Es preciso diseñar la propuesta de un plan como herramienta para aplicar las estrategias educativas y así elevar el índice académico de los estudiantes de la Escuela Estadal Bolivariana "Miguel Tobías Quiñonez" del municipio Barinas estado Barinas?; ¿Se hace necesario desarrollar las actividades del plan para consolidar el aprendizaje en los estudiantes de la Escuela Estadal Bolivariana "Miguel Tobías Quiñonez"?; ¿Es necesario valorar el progreso académico de los estudiantes, después de aplicada las estrategias educativas innovadoras, en la Escuela Estadal Bolivariana "Miguel Tobías Quiñonez"?

Es necesario impulsar cambios educativos planificados en el mejoramiento académico, alcanzando grandes cambios y condiciones personales y estudiantiles en los alumnos dando paso a un nuevo paradigma educativo; por ello esta contribuye a que en las casas de estudios se conviertan en lugares cómodos, alegres, activos, donde pongan en manifiesto y en práctica la creatividad, siendo los docentes facilitadores de estrategias educativas innovadoras para sus aprendizajes, no obviando los valores como pilares en la convivencia y ciudadanía.

Con el desarrollo de este trabajo investigativo se pretende influenciar al docente para que genere cambios de estrategias para un aprendizaje más dinamizado y significativo ofreciendo alternativas competentes al aprendizaje en los estudiantes, ya que a pesar de que estamos en el siglo XXI aún sigue imperando la monotonía en las aulas, la poca participación de los representantes, la falta de creatividad didáctica que muchas veces hace de la escuela y sus aulas un sitio aburrido para los niños, donde se precisa hacer un estudio que conlleve a un cambio de actitud por parte de los diferentes actores que involucre el accionar educativo. 


\section{Metodología}

\subsection{Naturaleza de la investigación}

El presente trabajo corresponde al paradigma cuantitativo, el cual según Hernández, Fernández y Baptista (2010): se caracteriza porque "ofrece la posibilidad de generalizar los resultados más ampliamente, otorga el control de los fenómenos y un punto de vista de conteo y magnitudes de estos" (pág. 81). De acuerdo con esto se puede decir, que permite el estudio de la realidad que envuelve desde lo perceptible o desde una visión que facilita medirla en términos reales, es decir, cuantificando sus características propias ubicándose en las ciencias fácticas.

\subsection{Tipo de la investigación}

Se enfoca en la categoría de proyecto factible, el cual según el Manual de trabajos de Grado de Especialización y Maestría y Tesis Doctorales de la Universidad Pedagógica Experimental Libertador (UPEL, 2016a), la define como aquella que: "consiste en la investigación, elaboración y desarrollo de una propuesta de un modelo operativo viable para solucionar problemas, requerimientos o necesidades de organizaciones o grupos sociales; puede referirse a la formulación de políticas, programas, tecnologías, métodos o procesos" (pág. 21); permitiendo por consiguiente proponer y ejecutar la propuesta para ver la veracidad y confiabilidad de sus resultados.

\subsection{Diseño de la investigación}

Se fundamenta en un estudio de campo, la cual consiste en obtener los datos directamente de la realidad.

En concordancia a lo expuesto, Arias (2006a), la define como:

Aquella que consiste en la recolección de datos directamente de los sujetos investigados o de la realidad donde ocurren los hechos (datos primarios), sin manipular o controlar variable alguna, es decir, el investigador obtiene la información, pero no 
altera las condiciones existentes. De allí su carácter de investigación no experimental (pág. 31).

Según este diseño, se fundamenta en tomar la información de primera mano, directa de la realidad sin manipulación alguna. En el mismo orden de ideas, el Manual de la Universidad Pedagógica Experimental Libertador (UPEL, 2016b), conceptualiza la investigación de campo como:

El análisis sistemático de problemas en la realidad, con el propósito bien sea de describirlos, interpretarlos, entender su naturaleza y factores constituyentes, explicar sus causas y efectos, o predecir su ocurrencia, haciendo uso de métodos característicos de cualquiera de los paradigmas o enfoques de investigación conocidos o en desarrollo (pág. 18).

De allí la necesidad en aplicar este diseño, el cual permite al investigador observar de manera directa la situación presentada, entendiendo las causas y los efectos que presenta el problema de la realidad misma y así recopilar los datos directos de la realidad.

De tal manera; que la investigación será enfatizada hacia el coordinador pedagógico y docentes de aula de educación primaria de la Escuela Estadal Bolivariana "Miguel Tobías Quiñonez", del Municipio y Estado Barinas, cuya característica principal, se centra en que se ejecutará en el ambiente de todos los participantes, en aras de presentar una propuesta orientada a la mejora del índice académico de los estudiantes.

\subsection{Fases de la investigación}

Para llevar a cabo la investigación, se tomará en cuenta la metodología propuesta en el Manual de la Universidad Pedagógica Experimental Libertador (UPEL, 2016c), la cual indica que:

El Proyecto Factible comprende las siguientes etapas generales: diagnóstico, planteamiento y fundamentación teórica de la propuesta; procedimiento metodológico, 
actividades y recursos necesarios para su ejecución; análisis y conclusiones sobre la viabilidad y realización del Proyecto; y en caso de su desarrollo, la ejecución de la propuesta y la evaluación tanto del proceso como de sus resultados. Los Trabajos de Grado de Especialización y de Maestría en la modalidad de Proyectos Factibles pueden llegar hasta la etapa de las conclusiones sobre su viabilidad, o pueden consistir en la ejecución y evaluación de Proyectos Factibles presentados y aprobados por otros estudiantes, para dar continuidad a líneas de investigación aplicada promovidas por el Instituto (pág. 21).

Por lo consiguiente, según las indicaciones del Manual de la Universidad Pedagógica Experimental Libertador (UPEL), este estudio se puede realizar en cuatro fases, ejecutando la propuesta con su consecuente evaluación del proyecto; por lo tanto, para la presente investigación se efectuará en cuatro fases:

Fase I: Diagnóstico, planteamiento y fundamentación teórica: Consistirá en establecer las necesidades de un grupo de docentes, mediante una investigación de campo para conocer la realidad que suscitan en sus aulas de clases en cuanto a la praxis docente de la Escuela Estadal Bolivariana "Miguel Tobías Quiñonez" parroquia Dominga Ortiz de Páez, del municipio Barinas, Estado Barinas, a través de la aplicación de estrategias pedagógicas, basadas en un estilo innovador, para optimizar el rendimiento académico de los estudiantes.

Fase II: Procedimiento metodológico: En esta área se presentará la forma de cómo se desarrollará la presente investigación para dar la posible solución al problema diseñándose el procedimiento, las técnicas, las variables, actividades, recursos, entre otros para su ejecución.

Fase III: Análisis y conclusiones: Una vez presentada las variables y evaluada las actividades se analizará e interpretará los datos y se procederá a organizar los resultados para comprobar la veracidad de la ejecución del 
trabajo investigativo y así disipar la problemática existente en dicha institución.

Fase IV: Ejecución y evaluación: consiste en el análisis de sus resultados y conclusiones sobre la viabilidad y realización del Proyecto; sin embargo, en esta investigación no se ha llegado a este punto o fase por lo que el trabajo aún se encuentra en avance.

\subsection{Población}

Al respecto, Tamayo y Tamayo (2003), la define como:

Totalidad de un fenómeno de estudio, incluye la totalidad de unidades de análisis o entidades de población que integran dicho fenómeno y que debe cuantificarse para un determinado estudio integrando conjunto $\mathrm{N}$ de entidades que participan de una determinada característica y se le denomina población por constituir la totalidad del fenómeno adscrito a un estudio o investigación (pág. 168).

La población que es el objeto de estudio estará constituida por siete (07) docentes, seis (06) de aula y un coordinador pedagógico de la Escuela Estadal Bolivariana "Miguel Tobías Quiñonez", parroquia Dominga Ortiz de Páez, del municipio Barinas, Estado Barinas.

Con respecto a la muestra, según Sampieri, Fernández y Baptista (2006a): "es un subgrupo de la población de interés sobre el cual se recolectarán datos, y que tiene que definirse o delimitarse de antemano con precisión" (pág. 273). La muestra de esta investigación es la misma población, estará conformada por siete $(07)$ docentes.

\subsection{Técnicas e instrumentos de recolección de datos}

Los instrumentos, son el método que se utiliza para reunir la información precisa e indispensable en la investigación, para ello se requiere diseñar formatos para los cuestionarios y guías para las entrevistas, entre otros, y el mismo se utiliza para obtener la información en el diagnóstico. 
Para Arias (2006b): "Las técnicas de recolección de datos son las distintas formas o maneras de obtener la información. Son ejemplos de técnicas; la observación directa, la encuesta en sus dos modalidades (entrevista o cuestionario), el análisis documental, análisis de contenido, etc." (pág. 38); lo cual se puede interpretar como la técnica que permite obtener la información para diagnosticar las necesidades o problemáticas.

En el presente trabajo se utilizará como técnica la encuesta, la cual se amparará en un cuestionario tipo escala de Likert, en virtud de facilitar el proceso de análisis e interpretación de los datos

\subsection{Cuestionario}

Desde esta perspectiva, Sampieri, Fernández y Baptista (2006b): expresan que "un cuestionario consiste en un conjunto de preguntas respecto de una o más variables a medir" (pág. 346); es decir; a través de este instrumento se plantean una serie de interrogantes que permitirán recabar la información precisa de los docentes sobre la temática que se aborda en este estudio.

\subsection{Validez}

Para la validez se usará la técnica del juicio de expertos la cual consiste en elegir un grupo de tres especialistas en las áreas de metodología, lenguaje y educación con especialidad en innovación, los cuales aplicaran los criterios pertinentes aprobando, desaprobando, o corrigiendo el instrumento en cuestión, esta valoración del juicio de expertos se hacen en virtud de que el cuestionario a aplicar tenga los estándares idóneos para la recolección de los datos, a fin de que el desarrollo de la investigación se ajuste con mayor proximidad posible a la realidad, y en consecuencia los resultados y conclusiones sean los más cónsonos con la investigación que aquí se plantea.

Según Sampieri, Fernández y Baptista (2006c): "La validez, en términos 
generales, se refiere al grado en que un instrumento realmente mide la variable que pretende medir. Por ejemplo, un instrumento válido para medir la inteligencia debe medir la inteligencia y no la memoria" (pág. 313).

\subsection{Análisis de los datos}

De acuerdo con Briones (2002), define:

El análisis, que se hace con base en un plan de análisis previamente preparado, comprende, básicamente, el estudio de los resultados estadísticos obtenidos con los datos. Son las respuestas cuantitativas a las preguntas y objetivos de la investigación: tablas de frecuencia, cuadros de doble entrada, asociaciones, correlaciones, etc. (pág. 49).

De esta manera luego de aplicar el instrumento definitivo a la muestra, se realizará la tabulación de los datos para determinar las frecuencias y porcentajes, que se representan en cuadros y gráficos a través del programa Microsoft Word; luego se procederá al análisis e interpretación mediante el uso de técnica estadística inferenciales para la interpretación de los resultados especialmente la descripción de los hechos de opinión de los docentes sujetos de investigación y se cruzara la información dada por ellos y se relacionará con las bases teóricas que permitirán dimensionar las incidencias de los hallazgos.

\section{Conclusión}

Se hace necesario que en las escuelas los docentes implementen en sus clases diarias, estrategias educativas innovadoras que logre captar la atención y el interés en los estudiantes, procurando generar cambios en cuanto a actitudes, aptitudes y competencias que favorezcan el crecimiento y desarrollo cognitivo y personal del alumno propiciando la oportunidad en que el estudiante descubra, sea creativo e inicie nuevas ideas en la construcción de su propio conocimiento. 
Se debe instaurar compromisos desde las instituciones donde el docente se planifique con estrategias educativas que abran paso a la invención e integración de los estudiantes fomentando brechas hacia la nueva teoría constructivista, siendo necesario para servir de cimiento al cambio en el contexto escolar.

En ese sentido se considera destacar la importancia que tiene la innovación dentro del conglomerado docente, para despertar en el alumno la inventiva y generar un ambiente de sinergia que conlleve a un ambiente óptimo para desarrollar el aprendizaje con los mejores resultados.

Se concluye que es factible ejecutar un plan de apoyo hacia el docente basadas en estrategias educativas innovadoras en función de sus intereses y necesidades para que el estudiante mejore el nivel de su rendimiento académico orientado en la integración, participación y producción activa del mismo.

\section{Referencias}

Arias, F. (2006a,b). El proyecto de investigación. 5ta Edición. Caracas, Venezuela: Editorial Episteme, C.A. / Orial Ediciones.

Briones, G. (2002). Metodología de la investigación cuantitativa en las ciencias sociales. Bogotá, Colombia: Editorial ICFES.

Nieto, S. (2008). Hacia una teoría sobre el rendimiento académico en enseñanza primaria a partir de la investigación empírica: Datos preliminares. Teoría de la Educación. Revista Interuniversitaria, 20, 249-274, ISSN: 1130-3743. Recuperado de:

http://revistas.usal.es/index.php/1130-3743/article/view/992

Hernández, R., Fernández, C., \& Baptista, P. (2010). Metodología de la investigación. México, D.F.: Editorial McGraw-Hill Interamericana Editores S.A.

Sampieri, R., Fernández, C., \& Baptista, P. (2006a,b,c). Metodología de la 
investigación. 4ta Edición, ISBN: 970-10-5753-8. México, D.F.: Editorial McGraw-Hill Interamericana Editores, S.A. de C.V.

Tamayo \& Tamayo, M. (2003). El proceso de la investigación científica. 4ta

Edición. México D.F.: Editorial Limusa, S.A. de C.V. Grupo Noriega Editores.

UPEL (2016a,b,c). Manual de trabajos de Grado de Especialización y Maestría y Tesis Doctorales. 5ta. Edición. Caracas, Venezuela: Fondo Editorial de la Universidad Pedagógica Experimental Libertador, UPELFEDUPEL. Recuperado de:

https://es.slideshare.net/mirnalitaguirrez/manual-upel-2016-1pdf 


\section{Lenny Yesenia Rojas Suárez}

e-mail: lennyrojas810@gmail.com

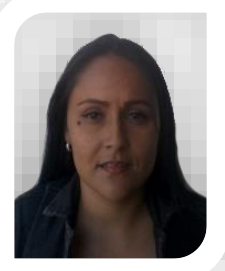

Nacida en Barinas, Venezuela. Me titule como Técnico Superior Universitario en Educación Integral en el Instituto Universitario de Tecnología Coronel Agustín Codazzi en julio del año 2007; en octubre del mismo año me inicie en el campo laboral como docente de aula de educación primaria; en el año 2009 retome mis estudios universitarios en la Universidad Nacional Experimental Simón Rodríguez, egresando en el año 2013 como Licenciada en Educación Integral; en octubre del año 2017, me asignaron la Dirección de la Escuela Estadal Bolivariana "Miguel Tobías Quiñonez", actualmente cumpliendo la función de supervisora del circuito educativo 4.39 del Eje Andino del Municipio Escolar Barinas.

El contenido de este manuscrito se difunde bajo una Licencia de Creative Commons ReconocimientoNoComercial-Compartirlgual 4.0 Internacional 